\title{
Protecting Against Damage from Refraction of High Power Microwaves in the DIII-D Tokamak
}

\author{
John Lohr ${ }^{1, *}$, Rigo Brambila ${ }^{l}$, Mirela Cengher ${ }^{1}$, Xi Chen ${ }^{l}$, Yuri Gorelov ${ }^{l}$, William Grosnickle ${ }^{1}$, Charles Moeller ${ }^{1}$, Dan \\ Ponce $^{1}$, Ron Prater ${ }^{1}$, Antonio Torrezan ${ }^{1}$, Max Austin ${ }^{2}$, Edward Doyle ${ }^{3}$, Xing Hu ${ }^{4}$, and Calvin Dormier ${ }^{4}$ \\ ${ }^{1}$ General Atomics, P.O. Box 85608, San Diego, California, USA \\ ${ }^{2}$ University of Texas-Austin, Austin, Texas, USA \\ ${ }^{3}$ University of California-Los Angeles, Los Angeles, California, USA \\ ${ }^{4}$ University of California-Davis, Davis, California, USA
}

\begin{abstract}
Several new protective systems are being installed on the DIII D tokamak to increase the safety margins for plasma operations with injected ECH power at densities approaching cutoff. Inadvertent overdense operation has previously resulted in reflection of an $\mathrm{rf}$ beam back into a launcher causing extensive arcing and melt damage on one waveguide line. Damage to microwave diagnostics, which are located on the same side of the tokamak as the ECH launchers, also has occurred. Developing a reliable microwave based interlock to protect the many vulnerable systems in DIII-D has proved to be difficult. Therefore, multiple protective steps have been taken to reduce the risk of damage in the future. Among these is a density interlock generated by the plasma control system, with setpoint determined by the ECH operators based on rf beam trajectories and plasma parameters. Also installed are enhanced video monitoring of the launchers, and an ambient light monitor on each of the waveguide systems, along with a Langmuir probe at the mouth of each launcher. Versatile rf monitors, measuring forward and reflected power in addition to the mode content of the rf beams, have been installed as the last miter bends in each waveguide line. As these systems are characterized, they are being incorporated in the interlock chains, which enable the ECH injection permits. The diagnostics most susceptible to damage from the ECH waves have also been fitted with a variety of protective devices including stripline filters, thin resonant notch filters tuned to the $110 \mathrm{GHz}$ injected microwave frequency, blazed grating filters and shutters. Calculations of $\mathrm{rf}$ beam trajectories in the plasmas are performed using the TORAY ray tracing code with input from kinetic profile diagnostics. Using these calculations, strike points for refracted beams on the vacuum vessel are calculated, which allows evaluation of the risk of damage to sensitive diagnostics and hardware.
\end{abstract}

\footnotetext{
* Corresponding author: 1ohr@fusion.gat.com
} 


\section{Introduction}

The Electron Cyclotron Resonance Heating (ECRH) system on the DIII-D tokamak uses gyrotron tubes generating nominally $1 \mathrm{MW}$ each at $110 \mathrm{GHz}$, which, after accounting for transmission losses, inject approximately $75 \%$ of the generated power into the tokamak. The system uses launchers with flexible steering so that the rf beams can intersect the second harmonic resonance nearly anywhere in the tokamak upper half plane, with toroidally oblique angles up to about $\pm 20^{\circ}$ from perpendicular for current drive. For low enough electron density, the rf propagates in nearly straight lines until being absorbed by the plasma at the second harmonic resonance. The right hand cutoff frequency is given for perpendicular incidence by

$$
\mathrm{f}_{\mathrm{R}}=0.5\left[\mathrm{f}_{\mathrm{ce}}+\left(\mathrm{f}_{\mathrm{ce}}{ }^{2}+4 \mathrm{f}_{\mathrm{pe}}{ }^{2}\right)^{0.5}\right] \text {, }
$$

which depends on the electron density through the electron plasma frequency term, where

$\mathrm{f}_{\mathrm{pe}}=9 \times 10^{3} \mathrm{n}_{\mathrm{e}}{ }^{0.5} \mathrm{~Hz}$, and $\mathrm{n}_{\mathrm{e}}$ is in $\mathrm{cm}^{-3}$.

If the density is high enough, the right hand cutoff layer can be at a frequency higher than the second harmonic over a part of the plasma, the specifics of which depend primarily on the density profile. Injected microwaves, which encounter the cutoff prior to being absorbed at the second harmonic, will be reflected or strongly refracted with little absorption. These reflected beams, at nearly full power, can enter diagnostic ports or strike windows, causing damage to components. This possibility could be a factor affecting the ITER operating space and poses risks for diagnostics on present day machines. On DIII-D, a number of diagnostics and ancillary hardware have been damaged by refracted and reflected $\mathrm{rf}$. This paper describes several of the situations which resulted in damage, and mitigating actions taken to prevent recurrence.

\section{Inadvertent Overdense Operation}

Overdense operation, resulting in refraction of the injected rf beams, can occur when the plasma density increases upon transition from L- to H-mode operation, when wall conditions are conducive to poor density control, with gas or pellet injection, when the poorly understood phenomenon of ECH density pumpout fails to control the density as desired, or under a number of other circumstances. When the rf fails to reach the second harmonic resonance owing to refraction, the results can be unpredictable, therefore all overdense operation is unintentional from an experimental point of view. As the density profile changes, rapid transitions from non-refractive to refractive behavior can result, with sweeping of the rf beams over a large fraction of the tokamak low field side wall, including into diagnostic ports, during the density increase. An example of extreme overdense operation is shown in Fig. 1.
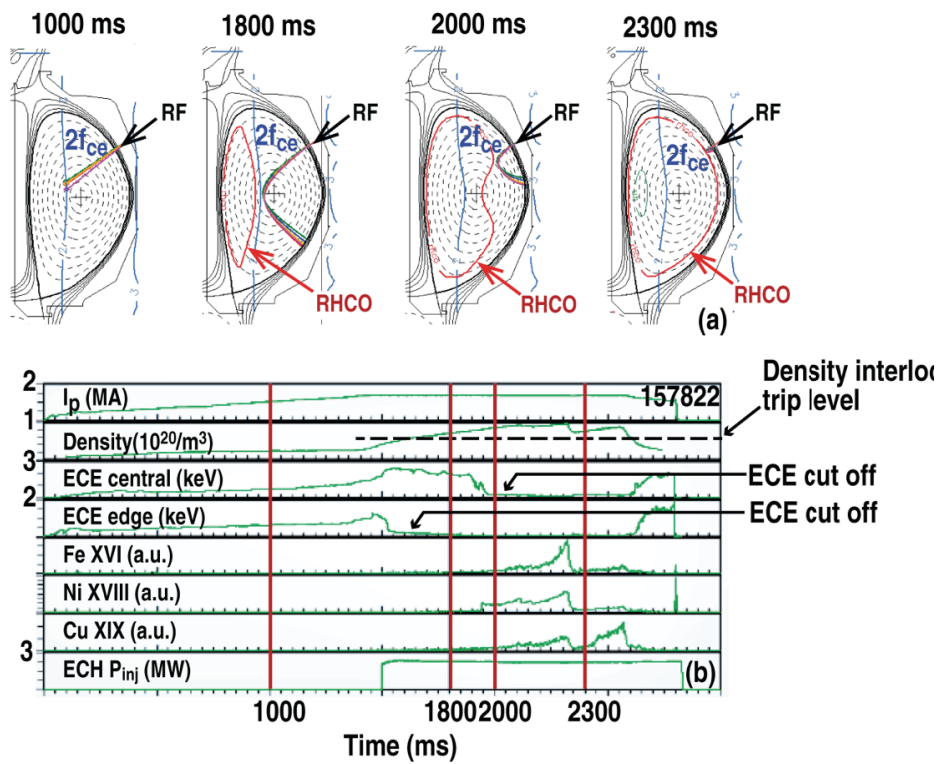

Fig. 1. This figure shows the progression of an extremely overdense plasma shot, on which the electron density by 2300 msec was high enough that the right hand cutoff frequency was immediately in front of the EC launchers, reflecting the rf power directly back into the launcher port. While the density increased, the rf beams swept across the low field side ports of the vacuum vessel. The complete cutoff of the ECE signals, a proxy for the existence of the cutoff layer, indicated that none of the injected rf power reached the second harmonic layer where it would have been absorbed. The label RHCO identifies the right hand cutoff layer. The metal lines seen spectroscopically indicate material from the $\mathrm{ECH}$ launchers was present in the plasma.

The ray tracing code TORAY was used to calculate the rf trajectories, using the plasma equilibrium for the shot and measured kinetic profiles normalized to a $\mathrm{j}(\mathrm{r})$ from the motional Stark effect diagnostic. In this case, the density increased until the right hand cutoff layer was directly in front of the launchers. An rf driven arc developed in one launcher, which caused melting of one of the two waveguides in the dual waveguide configuration. A Langmuir probe mounted at the output end of the waveguide was vaporized and the insulating ceramic beads on the bias wire were melted into glass. Because of the damage to the wires, the ECH permit was not removed by the Langmuir probe interlock. The damage is shown in Fig. 2. 

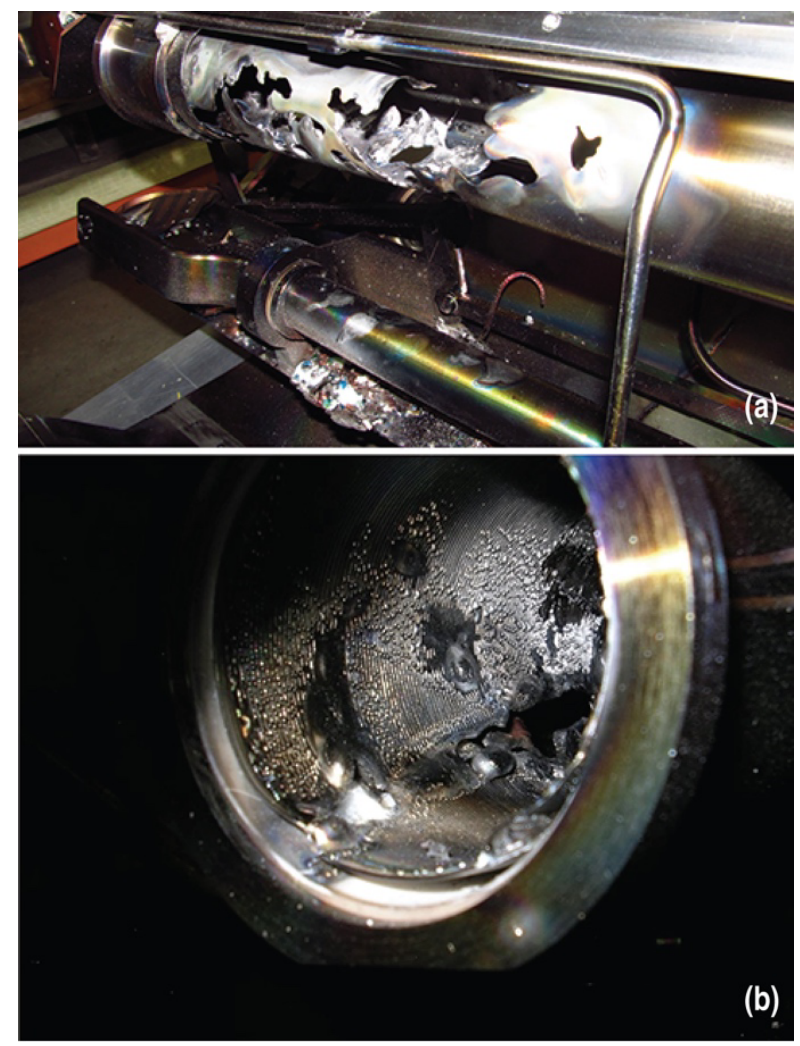

Fig. 2. The shot documented in Fig. 2 resulted in damage to one of the EC launchers, in which extensive melting of the waveguide and wires occurred. Fig (a) shows a view of the melted waveguide, and (b) is a photograph of the launch end of the waveguide. The damage was likely caused by rf heating of an arc, possibly where the third harmonic electron cyclotron resonance was present.

As can be seen from the progression of the beam trajectories in Fig. 1, any diagnostic on the low field side of the tokamak in the ECH launcher sector could have been subjected to rf heating as the beams swept across the ports. Microwave diagnostics, such as the ECE imaging diagnostic, located in the midplane port below the EC launchers, had suffered damage in the past and viewports had also been cracked, resulting in partial venting of the DIII-D vacuum vessel. It became clear that additional measures would be required to prevent damage to the installation under overdense conditions.

\section{Mitigation}

Mitigation of the risk of damage from ECH injection into the DIII-D tokamak has taken several forms. Administrative measures were developed, diagnostics were fitted with protective filters, and procedures were established to determine whether, given the operational parameters for the tokamak and the ECH system, there was a risk of damage. Additional diagnostics to provide risk assessment and protective fast response were installed.

A set of procedures was developed to clarify the allowable operational parameters. A density interlock was created based on the best available measurements as determined by the plasma control system. Each morning, this density limit was set automatically to a line average of $5.6 \times 10^{19} / \mathrm{m}^{3}$. This interlock line is shown on the time traces in Fig $1 \mathrm{~b}$. The first shot of the day, or the first shot after a major configuration change, would be required to be without ECH. Using the kinetic profiles from this shot, the ECH operators then calculate the rf beam trajectories using TORAY for the equilibrium and launcher aiming. Based on these calculations, the default density trip point could be changed to accommodate the experiment at as high a density as would be safe for the machine and diagnostics. A feature was added to the ray tracing code that calculates the trajectory of the rf rays for cases where $>10 \%$ of the injected rf power fails to be absorbed for any of the gyrotrons. This allows a determination of the likelihood that a particular set of plasma parameters and trajectories will result in damage, by identifying the ports into which power might be deposited. This calculation is shown in Fig. 3, for the shot in Fig. 1.
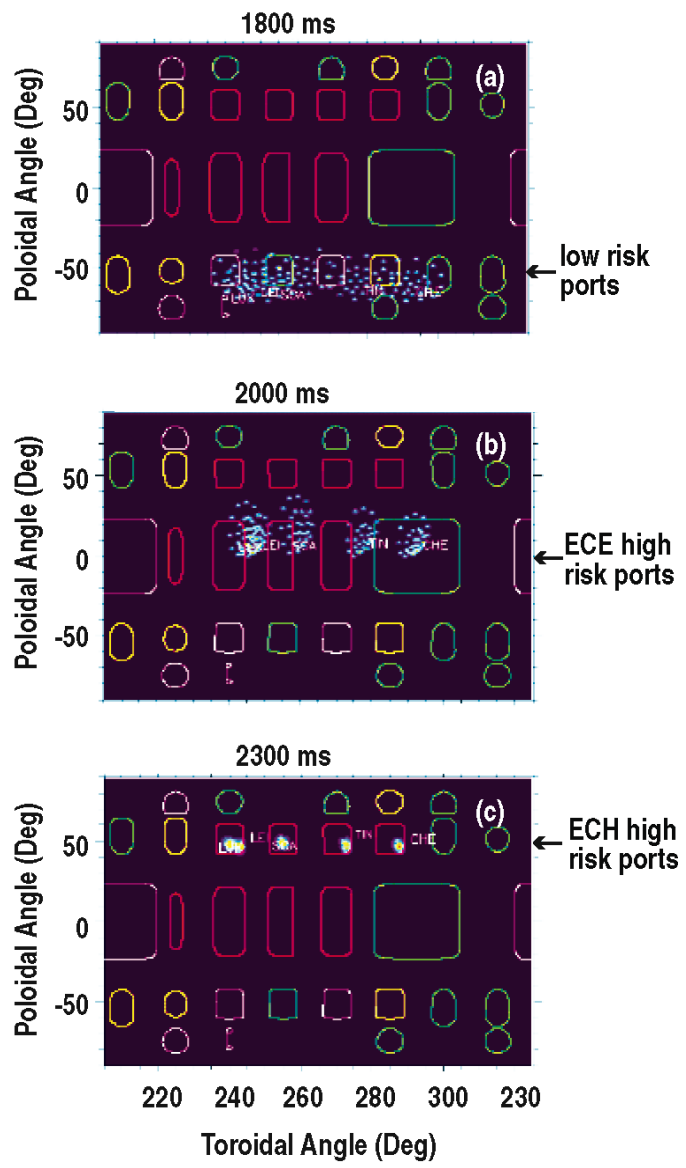

Fig. 3. In order to help assess the risk to the tokamak from $\mathrm{rf}$ refracted from the right hand cutoff layer, a code was written as an adjunct to TORAY, which calculates the points at which the refracted $\mathrm{rf}$ beams strike the vessel wall on the low field side. The plots show which ports will be struck for the plasma equilibrium and ECH launcher mirror steering in use using a multi-ray approximation to a free space Gaussian rf beam. Each rf beam is modeled by 100 rays. The strike points for each ray are plotted. 
In order to support these procedures, the accuracy of the diagnostic data, particularly the density profile, with Thomson scattering data verified by ECE cutoff and reflectometer data, and with equilibria normalized to $\mathrm{j}(\mathrm{r})$ measured by the Motional Stark Effect diagnostic, was

\subsection{Protective Hardware}

\subsubsection{Sniffer probes}

At several fusion research facilities attempts have been made to develop a detection system for stray $\mathrm{rf}$ power, which could serve as the basis for a protective interlock [1, 2]. At DIII-D, efforts were made to develop an rf detector, or "sniffer probe", which could be mounted at several locations around the tokamak vacuum vessel to help diagnose incomplete absorption of the injected $\mathrm{rf}$ and the potential for damage to occupants of ports. These tests did indicate when excessive rf was reflecting off the vessel walls, but showed that using the signals as a protective interlock resulted in an unacceptably large number of false trips, since we could not compensate for the polarization sensitivity of the detectors and the large signal variation arising from the angles with which the rf beams propagated in the vessel. One proposed solution to this problem has been to time-integrate the stray rf signal with a large cavity in which polarizations and trajectories would be isotropized prior to detection. These spheres worked in tests, but they occupied too much real estate in the close confines of the tokamak environment [3], at least on DIII-D. A new design for a sniffer probe has recently been presented [4] and this bolometer based probe is being evaluated on DIII-D. improved. Although the operating rules allowed a $10 \%$ safety factor for the density trip point to be set, with improved accuracy of the data used in the trajectory calculation, this safety factor cold be more accurately set to maximize the allowable parameter ranges.

Even if sniffers, which have a typical response time of $100 \mathrm{msec}$ for bolometers, can alert regarding low absorption of $\mathrm{ECH}$, they cannot respond quickly enough to protect diagnostics based on $\mathrm{mm}$ microwave technology employing diode detectors and mixers. Therefore protective measures must be installed on the diagnostics themselves. These steps have been taken on diagnostics systems on DIII-D.

\subsubsection{Filters}

The safest and most reliable protection against damage from $\mathrm{ECH}$ for diagnostics is for the diagnostics to be fitted with filters that reject the $110 \mathrm{GHz} \mathrm{ECH}$ microwave frequency. For receivers that use waveguide transmission lines, stripline filters can be used [5]. These are shaped conductors incorporated into a waveguide carrying the signal. The conductors are separated from a ground plane by a dielectric and the assembly is mounted in a housing connected to the waveguide. Depending on the shape of the conductors, bandpass, band reject, high or low pass filters can be designed using striplines. For microwaves in the 100 $\mathrm{GHz}$ range, high precision is required in the geometry of the structures, both in shape and thickness of the conductors. Stripline notch filters have been installed on the microwave reflectometers at DIII-D and provide about $-30 \mathrm{~dB}$ rejection.

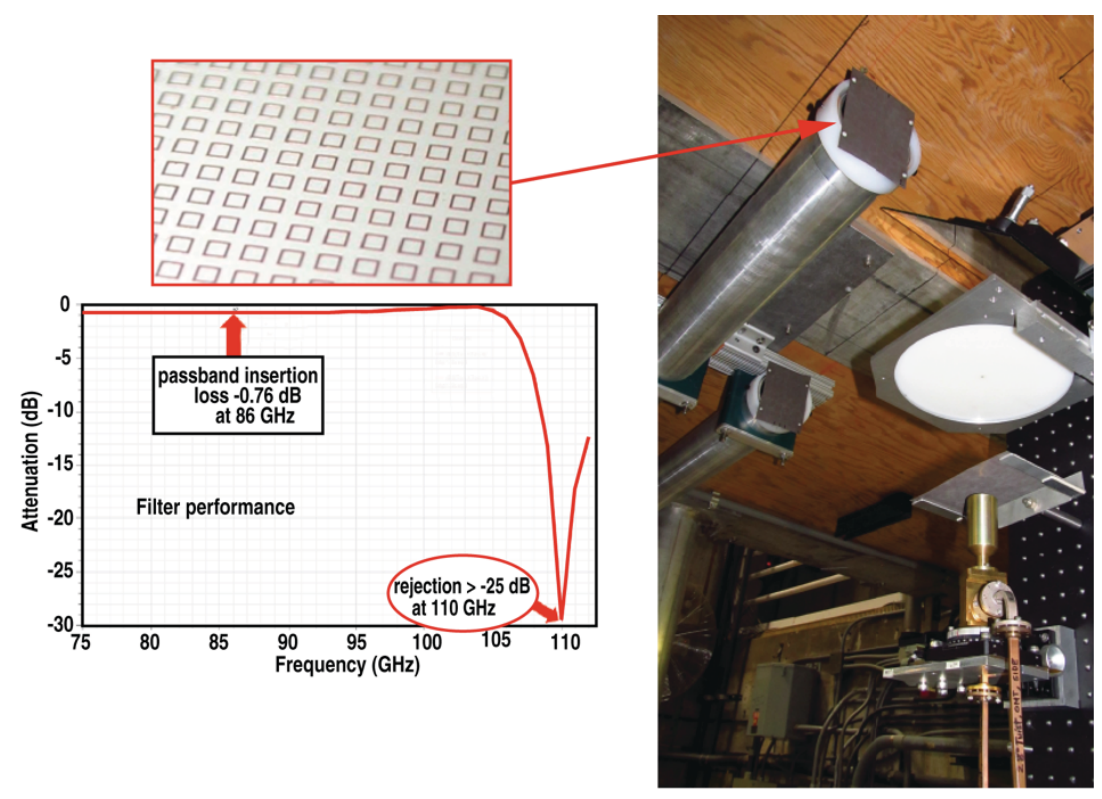

Fig. 4. Planar resonant cutoff filters produce a $-30 \mathrm{~dB}$ notch at the $110 \mathrm{GHz}$ gyrotron frequency, which can be increased to $-60 \mathrm{~dB}$ attenuation by stacking three of the filters in series. Insertion loss off-resonance is $<-1 \mathrm{~dB}$. The filters are produced by deposition of the repeated resonant structures onto a dielectric substrate using precise photolithographic techniques. The filters are easy to install and can be cut to shape using simple tools. 
A second class of notch filters uses an array of resonant structures applied to a dielectric substrate using printed circuit technology [6,7]. Although these filters, Fig. 4, are continuing to be improved, they can produce a notch width of about $5 \mathrm{GHz}$ at $-3 \mathrm{~dB}$, an insertion loss of less than $1 \mathrm{~dB}$ over a wide band, and a notch depth of $-30 \mathrm{~dB}$. By stacking up to 3 filter sheets in series with a few degree relative tilt, notch depths up to $-60 \mathrm{~dB}$ have been achieved. These can be combined with a dichroic plate providing high pass filtering to protect large arrays of microwave mixers and detectors used for imaging the plasma in the electron cyclotron radiation emitted from resonant surfaces, commonly the second harmonic of the electron cyclotron frequency. The filter sheets can be tilted up to 15 degrees without significantly affecting the transmission, making possible the series stacking mentioned above without developing standing waves due to reflections. The sheets can be cut with shears. In some cases both stripline and quasi-optical filters are used in series, providing about $-40 \mathrm{~dB}$ rejection.

\subsubsection{Light monitors}

A basic diagnostic, which has been in use on DIII-D for many years, is simple video imaging of the structure of the rf launchers seen through windows mounted in the launcher flange. The windows typically are sapphire in order to avoid microwave absorption, and provide a relatively good view of the launcher hardware through a wide angle lens. The video signals are observed by the ECH Operators, looking for excessive brightness or unexpected light persisting for seconds after the plasma shot, indicating incandescence from launcher components at high temperatures. The video images are archived on a multi-channel recorder allowing unusual events to be examined in more detail after the shot. In Fig. 5, an example of two video frames is shown, the first of which was taken during the ECH part of a plasma shot and the second, which showed persistent light about $5 \mathrm{sec}$ after the end of the plasma current from incandescence of melting launcher parts. In this case, the video monitors alone alerted the operators of a problem in the launcher.

In order to reduce the requirement for operator intervention, each waveguide line on the DIII-D system has been equipped with a visible light monitor to function in concert with the video system. The monitors are compact and mount directly on a sapphire viewing port with about the same field of view as the video cameras. Each light detector produces a modulated output, the frequency of which is proportional to the brightness of the detected light. This signal is routed to signal processing boards, which use f-V chips to produce an analog signal proportional to the detected light, but which is relatively immune to isolated light bursts and electrical noise. These signals are incorporated into an alarm system, which looks for light after the end of the plasma current.
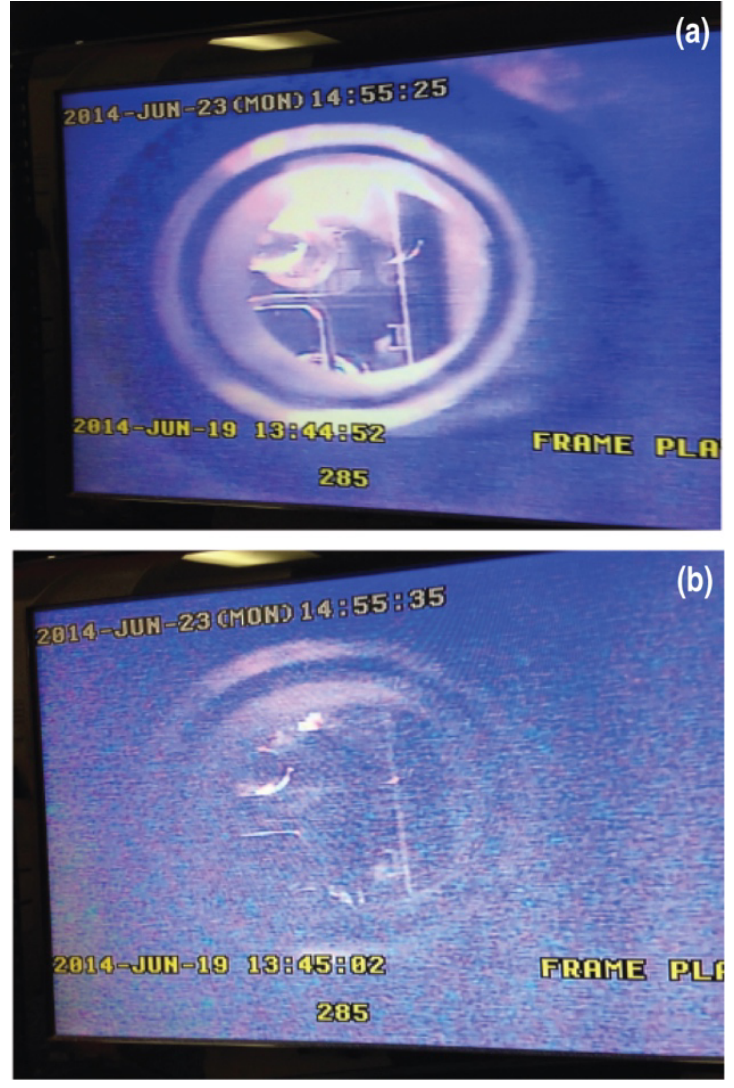

Fig. 5. These video images taken looking through windows in the EC launcher flanges provide a view of the launchers both in plasma light and in light from incandescent launcher assemblies long after the plasma current has ended. View (a) was taken during the plasma shot, and (b) was about $5 \mathrm{sec}$ after the end of the plasma, indicating the brightness of the incandescence well after the shot. Rather than rely on the ECH operators to take note of the persistent light following a shot, a set of visible light detectors were installed, which were interlocked to provide an alarm if the detected light persisted following the end of a plasma shot.

\subsubsection{Langmuir probes}

Presence of plasma in the vicinity of the launcher waveguides can be detected by Langmuir probes which are installed to the side of each waveguide mouth FIG. Each of these provides an interlock signal, which removes the $\mathrm{ECH}$ permit from the gyrotron connected to that waveguide. In some cases, ELMS, disruptions, pellet injection or instabilities can trip the Langmuir probe interlocks. In the highly overdense case described here, a flaw in the DIII-D launcher probe design was discovered. When the rf beams were reflected directly back into the launchers, the bias lead was immediately vaporized on one probe, melting the ceramic insulating beads and eliminating the probe signal being fed to the outside. The rf driven arc then caused the extensive waveguide melt damage seen in the photographs. To counteract this in the future, probe bias feed wires have all been placed in metal shields. It is hoped that now the probes will terminate the rf pulses prior to onset of damage. 


\subsubsection{Microwave detectors}

On all waveguide lines in the DIII-D system, the final miter bend in each waveguide line has now been instrumented to provide additional information, which can be used to interlock the rf generation. The advanced miter bends have been converted to 4 port devices with perforated mirrors that reflect most of the rf but pass a polarization insensitive signal at the $-75 \mathrm{~dB}$ level, which can be used with an orthomode transducer to measure the linear polarization of the $\mathrm{rf}$ beam. Using mode sensitive directional couplers, the miters can measure the content of several low order modes, $\mathrm{HE}_{1,1}, \mathrm{HE}_{2,1}$, or $\mathrm{TE}_{0,1}$, for example. The miter arms also can detect forward and reflected $\mathrm{rf}$ power, allowing an interlock to be based on the forward/reflected power ratio. These devices are now installed on all the ECH waveguide lines and are being set up to provide the interlock functions.

\section{Conclusions}

High power electron cyclotron resonant microwaves can be generated and directed with good precision into tokamak plasmas, heating plasma locally and driving current. Because the plasma density can be high enough that refraction and reflection can occur before the $\mathrm{rf}$ beams reach the resonance, where essentially complete absorption will occur, instead the rf rays can follow a curved path so the power can be absorbed at unintended flux surfaces intersecting with the rays and the resonance. If the refraction is strong, the rf can easily be reflected to unintended parts of the vacuum chamber, where it can be absorbed by diagnostics or other objects such as viewports, causing damage, venting of the vacuum vessel or even damage to the $\mathrm{ECH}$ system itself.

On DIII-D, after several cases of damage had occurred, mitigating measures were implemented. These included administrative procedures, installation of protection such as filters on the vulnerable diagnostics and equipment, and sensors on the $\mathrm{ECH}$ launchers to alert when densities have reached dangerous values. Additional measures are being considered, but in the meantime, no additional cases of damage have occurred anywhere on the tokamak from the ECH system.

\section{References}

1. F. Gandini, et.al.,Fusion Eng. Des., 56-57, 975 (2001).

2. H. Igami, et.al., Searching for O-X-B modeconversion window with monitoring of stray microwave radiation in LHD, Rev. Sci. Instruments, 77, 10E931 (2006).

3. M. Schubert, et.al., Monitoring millimeter wave stray radiation during ECRH operation at ASDEXUpgrade, Proc. 17th Joint Workshop on Electron Cyclotron Emission and Electron Cyclotron Resonance Heating, EPJ Web of Conferences 32, 02013 (2012).

4. H. Oosterbeek, Design and R\&D for the ITER Stray Radiation Bolometers, Proc. 19th Joint Workshop on Electron Cyclotron Emission and Electron Cyclotron Resonance Heating, Institute for Plasma Research, India (2016).

5. Terry Edwards and Michael Steer, Foundations for Microstrip Circuit Design, John Wiley and Sons, West Sussex, UK, ISBN:9781118936191 (2016).

6. H.J. van der Meiden, Rev. Sci. Instrum., 70, 6, 2861, (1999).

7. Z. Shen, et.al., Plasma and Fusion Research, 2, S1030 (2007).

This work is based upon work supported by the US Department of Energy, Office of Science, Office of Fusion Energy Sciences, using the DIII-D National Fusion Facility, a DOE Office of Science user facility under awards DE-FC02-04ER54698 ${ }^{1}$, DE-FG03-97ER54415², DE-FG0208ER54984 ${ }^{3}$, and DE-FG02-99ER54531 ${ }^{4}$. 